\title{
The Decision Analysis To Key Elements Of Marine Engineering Floating Pier Structure
}

\author{
Wenbai Liu ${ }^{1, a}$, Zhouxiang Wu ${ }^{1, b}$, Lin Yang $^{2, c}$ andQifei Fang ${ }^{1 d}$ \\ ${ }^{1}$ College of Ocean Environment and Engineering, Shanghai Maritime University, 1550 Pudong \\ Avenue, Shanghai, 200135, China \\ ${ }^{2}$ Guangxi Transportation Research \& Consulting Co, Ltd,No.6 Hi Tech Zone, Nanning, Guangxi, \\ 530007, China \\ aliuwb8848@163.com,x371685880@sina.com,cyuyl999@163.com, dfangqifei05@163.com
}

\begin{abstract}
Keywords: VLFS,Floating Pier,Grid Structure, Key Elements, matrix relative condition number Abstract. Some components are not important when examining the structural integrity, but their destruction can lead to the continuous collapse of the overall structure. Such components are called key Elements. In this chapter, the determination of the key elements of a marine engineering floating .pier structure is carried out by using the judgment method of the overall stiffness matrix relative condition number based on the structural balance equation. The components at the four corners of the structure have a high degree of importance and are the key elements of the structure.
\end{abstract}

\section{Introduction}

The key elements is the element whose damage will cause the overall structure to become unstable and collapse. The marine engineering floating pier structure (VLFS) studied in this paper belongs to the large-span spatial network structure. It consists of a number of standardized rods and nodes and other basic components to form a spatial high-order statically determinate network structure according to the pattern of the grid. This type of structure has a high degree of redundancy at the nodes where the rods meet, and the rods within the connection range are prone to adverse conditions such as stress concentration and buckling instability. Therefore, for the marine engineering floating pier structure, any one of the rods may become a key element of the structure.

\section{The Method of Judging the Condition Number of Whole Stiffness Matrix}

Based on the determination of the key elements of structural redundancy, ductility, and structural robustness (to avoid collapse of structures for the purpose of overall structural safety), experts and scholars have proposed them from the aspects of vulnerability theory, stiffness theory, and energy perspectives. The corresponding judgment method ${ }^{[1,2]}$ has wide applicability and engineering applicability. Structural calculations can eventually be turned into mathematical problems for analysis. Among them, the overall stiffness matrix condition number determination method proposed by Zhan Hailei et al. (2014) ${ }^{[3]}$ gives a simple way to predict whether structural elements failure will cause continuous collapse of the whole. The critical element determination method for the condition number of the overall stiffness matrix assumes that the structure meets the ideal elasticity and small deformation theory, and the overall structure satisfies the equilibrium equation.

$[\mathrm{K}]\{\delta\}=\{\mathrm{F}\}$

Where $[\mathrm{K}]$ is the overall stiffness matrix of the structure, $\{\delta\}$ is the array of displacement components of the overall structure, and $\{\mathrm{F}\}$ is the array of nodal forces of the overall structure.

Based on the overall stiffness matrix $[K]$, the relative condition number $R(K)$ of the defined structure is the ratio of the condition number of the overall stiffness matrix of the structure after the local failure to the condition of the overall stiffness matrix of the original structure ${ }^{[4]}$ :

$\mathrm{R}(\mathrm{K}) \mathrm{i}=\frac{\operatorname{CondK}_{\mathrm{i}}}{\mathrm{CondK}_{\mathrm{n}}}, \mathrm{i}=0,1,2 \ldots$

From equation (2), it can be seen that the greater the number of relative conditions of the new structure that has been destroyed, the more important the structural element that has been destroyed. The element $\mathrm{i}$ is the key element of the structure. 


\section{The Overall Stiffness Matrix Conditional Number Method for Judging the Key Element of Floating Grid}

Analysis object selection. Select the center of the super-large floating structure for analysis. Take half the height of the three-layer grid $\mathrm{H}=18 \mathrm{~m}$, the angle between the web and the horizontal plane $\alpha=60^{\circ}$, the diameter of the ball joint $\mathrm{D} 1=6.5 \mathrm{~m}$, and the diameter of the rod $\mathrm{D} 2=2.13 \mathrm{~m}$, ball joint and rod wall thickness $t=25 \mathrm{~mm}$. As shown in Fig.2, due to the large number of poles, and mainly analyzing the influence of the components in each quadrangular pyramid system on the mechanical properties of the overall structure, the analysis object is simplified here: In the xz plane, the poles are erected according to the grid structure. The arrangement of parts, with a quadrangular pyramid as the basic element, simplifies the analysis of the three-dimensional network structure into a plane grid for analysis; the ball node is simplified as a node in the stiffness matrix analysis, regardless of its geometric size; according to the symmetry of the structure, Select half of the structure for analysis, as shown in Fig.3.

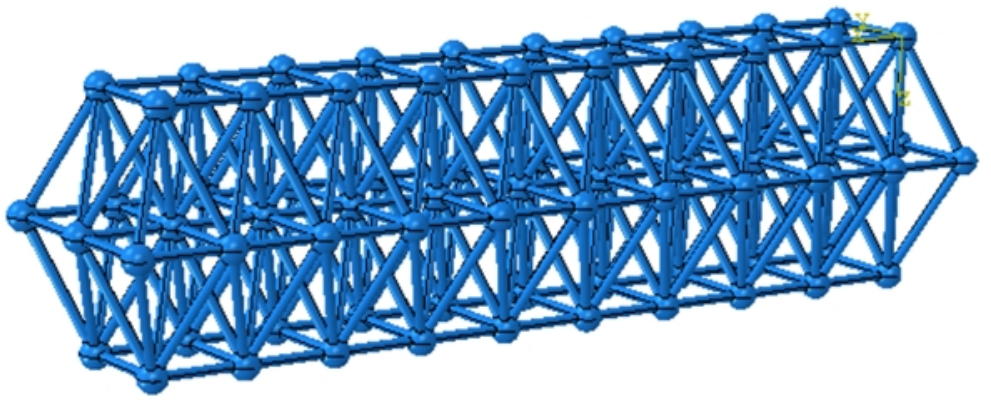

Fig.1 Marine engineering floating pier structure

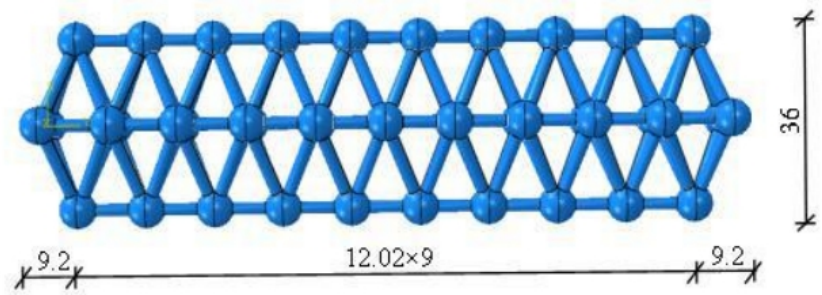

Fig.2 Elevation diagram of three-story square pyramid grid structure of ocean floating structure

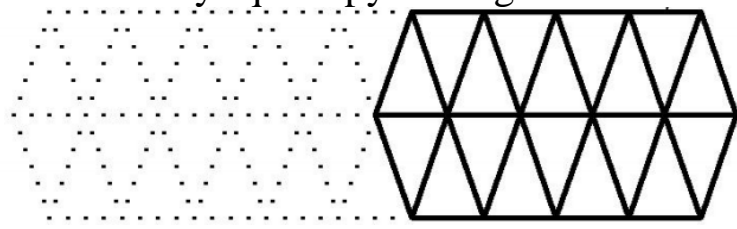

Fig.3 Object selection for analysis of key elements of a truss in a marine floating structure

Calculation of element stiffness matrix. The study object shown in Fig. 3 is placed in the plane $x-y$ coordinate system, as shown in Fig.4. The structure is divided into 18 elements from [1] to [18] according to the plane position of each triangle cone, and the nodes of each element are numbered 1 , $2,3 \ldots 16$. Take the triangle element base length $12.02 \mathrm{~m}=2 \mathrm{~A}$, high take $10.41 \mathrm{~m}=\mathrm{B}$, triangular elements area $\mathrm{S}=15.642 \mathrm{~m}$, rod thickness take $\mathrm{h}=25 \mathrm{~mm}$, elastic modulus $\mathrm{E}=2.111 \times 10 \mathrm{~Pa}$, Poisson's ratio $\lambda=0.3$, Structural forces are in static equilibrium.

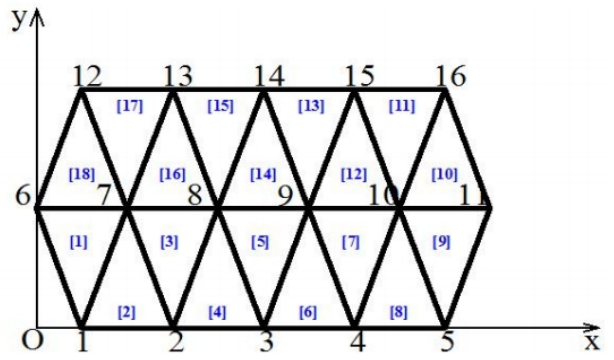

Fig.4 Grid structure in a planar rectangular coordinate system 
The overall stiffness matrix of the analysis object of the grid structure in the planar rectangular coordinate system in Fig.3 is obtained by combining 18 subunit stiffness matrices, so the element stiffness matrix of all the elements should be obtained first: ${ }^{[5]}$

For each, there is a balance equation

$[\mathrm{K}]\{\delta\}^{\mathrm{e}}=\{\mathrm{F}\}^{\mathrm{e}}$

For a triangular cell stiffness matrix with three nodes, the write component block is

$[\mathrm{K}]=\left[\begin{array}{ccc}k_{i i} & k_{i j} & k_{i m} \\ k_{j i} & k_{j j} & k_{j m} \\ k_{m i} & k_{m j} & k_{m m}\end{array}\right]$

The size of each sub-matrix in the above formula is $2 \mathrm{X} 2$. Here we study the problem of plane stress.

$\left[\mathrm{K}_{\mathrm{rs}}\right]=\frac{E A}{4\left(1-\lambda^{2}\right) s}\left[\begin{array}{ll}b_{r} b_{s}+\frac{1-\lambda}{2} c_{r} c_{s} & \lambda b_{r} b_{s}+\frac{1-\lambda}{2} c_{r} b_{s} \\ \lambda c_{r} b_{s}+\frac{1-\lambda}{2} b_{r} c_{s} & c_{r} c_{s}+\frac{1-\lambda}{2} b_{r} b_{s}\end{array}\right]$

among them, $b_{i}=y_{i}-y_{m^{\prime}} c_{i}=-x_{j}+x_{m}(\mathrm{I}, \mathrm{j}, \mathrm{m})$

The element stiffness matrix is calculated using element [1] as an example. The specific steps are as follows:

The first step, the list calculation b, c (according to counterclockwise selection node), see Table 1

Table 1 Element stiffness matrix parameter calculation table

\begin{tabular}{|c|c|c|c|c|}
\hline node parameter & $\mathrm{x}$ & $\mathrm{y}$ & $\mathrm{b}$ & $\mathrm{c}$ \\
\hline 1 & $\mathrm{~A}$ & 0 & 0 & $-2 \mathrm{~A}$ \\
\hline 7 & $2 \mathrm{~A}$ & $\mathrm{~B}$ & $\mathrm{~B}$ & $\mathrm{~A}$ \\
\hline 6 & 0 & $\mathrm{~B}$ & $-\mathrm{B}$ & $\mathrm{A}$ \\
\hline
\end{tabular}

The second step is to find the sub-matrix [ $\left.\mathrm{k}_{\mathrm{rs}}\right]$, which is obtained by formula (5). The meanings and values of each symbol in the formula are defined at the beginning of this section.

$\left[\mathrm{K}_{11}\right]=\frac{E A}{4\left(1-\lambda^{2}\right) S}\left[\begin{array}{cc}\frac{1-\lambda}{2} 4 A^{2} & 0 \\ 0 & 4 A^{2}\end{array}\right]$
$\left[\mathrm{K}_{77}\right]=\frac{E \hbar}{4\left(1-\lambda^{2}\right) S}\left[\begin{array}{cc}B^{2}+\frac{1-\lambda}{2} A^{2} & \lambda A B+\frac{1-\lambda}{2} A B \\ \lambda A B+\frac{1-\lambda}{2} A B & A^{2}+\frac{1-\lambda}{2} B^{2}\end{array}\right]$
$\left[\mathrm{K}_{66}\right]=\frac{E s}{4\left(1-\lambda^{2}\right) S}\left[\begin{array}{cc}B^{2}+\frac{1-\lambda}{2} A^{2} & -\lambda A B-\frac{1-\lambda}{2} A B \\ -\lambda A B-\frac{1-\lambda}{2} A B & A^{2}+\frac{1-\lambda}{2} B^{2}\end{array}\right]$
$\left[\mathrm{K}_{17}\right]=\frac{E \hbar}{4\left(1-\lambda^{2}\right) S}\left[\begin{array}{cc}\frac{1-\lambda}{2}\left(-2 A^{2}\right) & \frac{1-\lambda}{2}(-2 A B) \\ -2 \lambda A B & -2 A^{2}\end{array}\right]=\left[\mathrm{K}_{71}\right]$
$\left[\mathrm{K}_{16}\right]=\frac{E \hbar}{4\left(1-\lambda^{2}\right) S}\left[\begin{array}{cc}\frac{1-\lambda}{2}\left(-2 A^{2}\right) & \left.\frac{1-\lambda}{2}(2 A B)\right]=\left[\mathrm{K}_{61}\right] \\ 2 \lambda A B & -2 A^{2}\end{array}\right]$
$\left[\mathrm{K}_{76}\right]=\frac{E \hbar}{4\left(1-\lambda^{2}\right) S}\left[\begin{array}{cc}-B^{2}+\frac{1-\lambda}{2} A^{2} & \lambda A B-\frac{1-\lambda}{2} A B \\ -\lambda A B+\frac{1-\lambda}{2} A B & A^{2}-\frac{1-\lambda}{2} B^{2}\end{array}\right]=\left[\mathrm{K}_{67}\right]$

The remaining 17 triangular elements are calculated according to this method and will not be described in detail here.

Synthesis of Structure Stiffness Matrix. Combine all element balance equations to form the overall equilibrium equation of the structure

$[\mathrm{K}]\{\delta\}=\{\mathrm{F}\}$

The overall stiffness matrix $[\mathrm{K}]_{2 \mathrm{n} \times 2 \mathrm{n}}$ is assembled from the element stiffness matrix. The assembly method is to place the single rigid[ $\left.\mathrm{K}_{\mathrm{rs}}\right]_{2 \times 2}$ in the rth row and the sth column of the overall stiffness matrix, and the overall stiffness matrix assembled is assembled. As shown by the following formula: [6] 
$[\mathrm{K}]_{2 \mathrm{n} \times 2 \mathrm{n}}=\left[\begin{array}{ccccccc}\ldots & \ldots & \ldots & \ldots & \ldots & \ldots & \ldots \\ \ldots & k_{i i} & \ldots & k_{i j} & \ldots & k_{i m} & \ldots \\ \ldots & \ldots & \ldots & \ldots & \ldots & \ldots & \ldots \\ \ldots & k_{j i} & \ldots & k_{j j} & \ldots & k_{j m} & \ldots \\ \ldots & \ldots & \ldots & \ldots & \ldots & \ldots & \ldots \\ \ldots & k_{m i} & \ldots & k_{m j} & \ldots & k_{m m} & \ldots \\ \ldots & \ldots & \ldots & \ldots & \ldots & \ldots & \ldots\end{array}\right] \quad(6)$

Here, $\mathrm{n}=16$, forming a $32 \times 32$ overall stiffness matrix for the overall structure

$$
\left[\mathrm{K}_{0}\right]=\left[\begin{array}{ccccccccc}
171.58 & 40.66 & -95.73 & -3.13 & 0 & 0 & \ldots & 0 & 0 \\
40.66 & 218.53 & 3.13 & -1.81 & 0 & 0 & \ldots & 0 & 0 \\
-95.73 & 3.13 & 292.58 & 0 & -95.73 & -3.13 & \ldots & 0 & 0 \\
-3.13 & -1.81 & 0 & 292.58 & 3.13 & -1.81 & \ldots & 0 & 0 \\
0 & 0 & -95.73 & 3.13 & 292.58 & 0 & \ldots & 0 & 0 \\
0 & 0 & -3.13 & -1.81 & 0 & 292.58 & \ldots & 0 & 0 \\
\ldots & \ldots & \ldots & \ldots & \ldots & \ldots & \ldots & \ldots & \ldots \\
0 & 0 & 0 & 0 & 0 & 0 & \ldots & 171.58 & 40.66 \\
0 & 0 & 0 & 0 & 0 & 0 & \ldots & 40.66 & 218.53
\end{array}\right]
$$

Observing the overall structure and the position of each element, it can be seen that elements [2][4][6][8][11][13][15][17] involve all the horizontal rods of a three-layered quadrangular pyramidal grid structure and almost For all webs (except for the edges of the structure), the triangular elements of the web are [1][9][10][18]. Here, the above elements[1][2][4][6][8][9][10][11][13][15][17][18] are successively removed from the overall structure. According to the above steps, the overall stiffness matrix $[\mathrm{K}]$ of the corresponding element is calculated.

Matlab solves the stiffness matrix condition number. In Matlab, the function statement called by the condition number of matrix $\mathrm{K}$ is $\mathrm{Cond}(\mathrm{K})$ and the corresponding relative condition number $\mathrm{R}(\mathrm{K})$, $\mathrm{R}(\mathrm{K})=\frac{\text { CondK }}{\mathrm{CondK}_{\mathrm{n}}}$, as shown in Table 2 :

Table 2 Corresponding condition number and relative condition number of each structure

\begin{tabular}{|c|c|c|}
\hline Extraction elements & Cond $(\mathrm{K})$ & $\mathrm{R}(\mathrm{K})$ \\
\hline Original structure & 113.50 & 1.000 \\
\hline$[1]$ & 231.44 & 2.039 \\
\hline$[2]$ & 152.56 & 1.3441 \\
\hline$[4]$ & 128.69 & 1.1338 \\
\hline$[6]$ & 116.79 & 1.0290 \\
\hline$[8]$ & 131.09 & 1.1550 \\
\hline$[9]$ & 139.36 & 1.2278 \\
\hline$[10]$ & 142.29 & 1.2537 \\
\hline$[11]$ & 134.11 & 1.1816 \\
\hline$[13]$ & 113.53 & 1.0003 \\
\hline$[15]$ & 114.17 & 1.0059 \\
\hline$[17]$ & 118.99 & 1.0484 \\
\hline$[18]$ & 128.09 & 1.2166 \\
\hline
\end{tabular}

Critical element determination. The relative condition number $\mathrm{R}(\mathrm{K})$ obtained from the analysis reveals that the number of relative conditions after removing the elements ([1] and [18], [9] and [10]) at the edge of the grid, length direction is larger than the others. Explain that the failures of elements [1], [9], [10] and [18] are most likely to lead to the collapse of a truss structure in the center of an oceanic super-floating structure; the maximum number of relative conditions $R(K) \max$ is After element [1] is generated; and when the element ([4][6][13][15]) near the axis of symmetry of the analysis object is extracted, its relative condition number $\mathrm{R}(\mathrm{K})$ is almost the same as the structure prototype, indicating that these Damage to the location of the element as a whole has little effect. 


\section{Conclusion}

From the calculation results, we can see that for the marine engineering floating pier structure studied in this study, the components in the middle part of the structure have a relatively small impact on the overall structure; the structural components at the four corners of the structure have a high degree of importance and are the key to the structure element. In the design stage, the three-layer square pyramid structure at the corners of the structure can be reinforced.

\section{Acknowledgements}

This work was financially supported by National Natural Science Foundation of China (51078228), National Marine public welfare industry special research funding project (201105024-5)

\section{References}

[1] Cai Jianguo, Wang Feng, Han Yunlong and others. Long-span spatial structure important components evaluation practical method [J]. Journal of Hunan University(Natural Science), 2011, 38(3):7-11.

[2] Ye Lieping, Cheng Guangyi, Lu Xinzheng et al. On the structural robustness [J]. Building Structure, 2008, 6:11-15.

[3] Y. Mishing, in: Diffusion Processes in Advanced Technological Materials, edtied by D. Gupta Noyes Publications/William Andrew Publising, Norwich, NY (2004), in press.

[3] Zhan Hailei, Xiao Nan. Global and local decision methods for key elements of pole structure[J]. Space Structure, 2014, 20(1):58-63.

[4] Zhan Hailei. Spatial structure key element search method [D]. Zhejiang: Zhejiang University, 2014.

[5] Wang Yuanhan, Li Lijuan, Li Yinping. Finite Element Method Foundation and Programming [M]. Guangzhou: South China University of Technology Press, 2002, 8:8-14.

[6] Yang Mingsheng, Xiong Xiwen, Lin Jianhua. MATLAB Basics and Mathematical Software [M]. Dalian: Dalian University of Technology Press, 2003. 